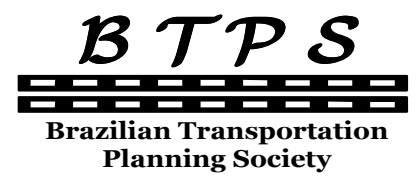
Journal of Transport Literature
Vol. 8, n. 2, pp. 221-243, Apr. 2014
Research Directory

JTL|RELIT

www.transport-literature.org ISSN 2238-1031

\title{
Modelo de previsión de la progresión del área de fisuras en pavimentos asfálticos
}

[A model to predict cracking progression in hot-mix asphalt pavements]

\author{
Sérgio Pacífico Soncim*, José Leomar Fernandes Junior \\ Federal University of Bahia - Brazil, University of São Paulo - Brazil
}

Submitted 3 Dec 2012; received in revised form 26 Mar 2013; accepted 28 Apr 2013

\begin{abstract}
Resumen
Este estudio presenta el desarrollo de un modelo para la previsión de la progresión del área de fisuras en pavimentos con concreto asfáltico caliente, con informaciones de un banco de datos de gerencia de carreteras fornecido por el Departamento de Infra-estructura de Transportes del Estado de Bahia, colectados en 2004. Fue desarrollado un planeamiento factorial con el objetivo de organizar los datos disponibles para el posterior análisis estadístico. Los factores considerados fueron la edad del pavimento, el tráfico y la pluviometría. Fue realizado un estudio comparativo entre el modelo desarrollado y otros modelos existentes, a través de la evaluación del ajuste de los valores previstos por los mismos con los valores observados en campo, teniendo como base su evolución a lo largo del tiempo. El modelo desarrollado en este trabajo, en comparación con los modelos de Marcon (1996) y Paterson (1987), presentó mejor correlación entre los valores observados y los previstos, lo que demuestra evidencia del la validez de su utilización en análisis de redes carreteras constituidas por revestimientos asfálticos.
\end{abstract}

Key words: sistemas de gerencia de pavimentos, modelos de desempeño, defectos, fisuras.

\section{Abstract}

This work presents the development of a model to predict cracking progression in hot-mix asphalt pavements, based on information obtained from a database maintained by State of Bahia Department of Transportation, with data collected in 2004. A factorial design was performed to arrange the data available in order to facilitate the statistical analysis. The factors considered were age of the pavement, traffic volume and rainfall. The model developed in this work to predict cracking progression was compared to other existing models through the evaluation of the fit to the observed data, based on its evolution over time. The performance model developed in this work was compared to performance models developed by Marcon (1996) and Paterson (1987) presented the best results, in an evidence of its applicability to other highway networks.

Palavras-Chave: pavements management systems, performance models, distresses, cracking.

*Email: sergiops@ufba.br.

\section{Recommended Citation}

Soncim, S. P. and Fernandes Jr, J. L. (2014) Modelo de previsión de la progresión del área de fisuras en pavimentos asfálticos. Journal of Transport Literature, vol. 8, n. 2, pp. 221-243.

- JTL/RELIT is a fully electronic, peer-reviewed, open access, international journal focused on emerging transport markets and published by BPTS - Brazilian Transport Planning Society. Website www.transport-literature.org. ISSN 2238-1031. 


\section{Introducción}

El objetivo de este estudio fue desarrollar un modelo para la previsión de la progresión del área de fisuras en pavimentos construidos con concreto asfáltico caliente, según las características de la malla carretera del Estado de Bahía, Brasil. El Estado de Bahía está situado en una región de clima tropical. Su extensión territorial lo convierte en el quinto estado más grande de Brasil. La malla carretera estatal bahiana tiene más de $20.000 \mathrm{~km}$ de caminos pavimentados. Debido a su considerable extensión, el Estado de Bahía presenta notables variaciones en los índices de precipitación pluviométrica, en su topografía y en su vegetación.

El desempeño de un pavimento puede ser definido como la variación de la serviciabilidad que el pavimento ofrece a sus usuarios, con el transcurso del tiempo o después de un número de aplicaciones de cargas de tráfico, es decir, el histórico de la serviciabilidad brindada al usuario (Carey y Irick, 1960). La serviciabilidad se define como la capacidad de un pavimento en satisfacer la demanda del tráfico con confort, seguridad y economía.

Los modelos de previsión de desempeño utilizados para auxiliar en los procesos de decisión son útiles cuando son capaces de explicar el proceso de deterioro de los pavimentos. La estimativa del desgaste y su evolución son importantes en la determinación de la vida de servicio del pavimento, definida como el periodo comprendido entre el inicio de la aplicación de cargas de tráfico y una intervención de rehabilitación.

Los modelos de previsión de desempeño deben exponer las condiciones sobre las cuales son aplicados y deben ser formulados a partir de datos locales. Brasil representa un excelente ejemplo de esa necesidad, ya que posee condiciones climáticas diversificadas y una gran variedad de suelos (Queiroz, 1984). La evaluación del desempeño de las carreteras es esencial para un Sistema de Gerenciamiento de Pavimentos (SGP), tanto a nivel de red como a nivel de proyecto (Lytton, 1987). A nivel de red, la previsión del desempeño del pavimento es importante para planificación, priorización de proyectos y asignación de recursos. A nivel proyecto es importante para definir actividades de mantenimiento y rehabilitación, además exige una mayor precisión en las previsiones, ya que a nivel de red son usadas para obtener 
una estimativa de las necesidades totales de mantenimiento y rehabilitación, con el propósito de programación de inversiones (Haas y Hudson, 1978).

Para desarrollar el modelo fueron utilizadas las informaciones de un banco de datos de gerencia de carreteras facilitado por el Departamento de Infraestructura de Transportes del Estado de Bahía (DERBA), colectados en 2004. Vale notar que los pavimentos, construidos con asfalto caliente, no habían sufrido ninguna intervención de mantenimiento antes del 2004.

Fue elaborado un programa factorial con el objetivo de organizar los datos disponibles en el banco de datos del DERBA de tal forma que, las matrices factoriales facilitasen su posterior análisis estadístico y el desarrollo del modelo de desempeño de los pavimentos. Los factores considerados fueron la edad del pavimento, el tráfico y la pluviometría. Para la ejecución de las matrices factoriales fueron adoptados dos niveles para cada factor.

Para evaluar la significancia de los factores y definir los parámetros del modelo, los datos fueron analizados estadísticamente a través del Análisis Exploratorio de Datos (AED) y del Análisis de Varianza (ANOVA). El AED presenta un abordaje de simple aplicación y de gran importancia en la fase inicial del análisis de un experimento factorial. Utiliza una gran variedad de técnicas, en su mayoría gráficas, para maximizar la percepción dentro de un grupo de datos.

El AED se mostró eficaz en la determinación de los efectos de los factores y sus interacciones y sus resultados fueron corroborados por los resultados obtenidos a través del ANOVA. El modelo de previsión desarrollado en este trabajo, en comparación con los modelos de Marcon (1996) y Paterson (1987), presentó mejor correlación entre los valores observados y los previstos.

El resto del documento está organizado como sigue: en la sección 1 se presenta una revisión de la literatura sobre modelos de previsión de la progresión del área de fisuras desarrollados sobre la base de datos de diferentes regiones de Brasil. Una descripción de los datos y planificación factorial se presentan en la sección 2. La Formulación del modelo de previsión de la progresión del área de fisuras se presentan en la sección 3 y la análisis de los efectos de los factores en la sección 4. Una Análisis comparativo con otros modelos de desempeño se 
presenta en la sección 5 y en la última sección se presentan las conclusiones y sugerencias para estudios futuros.

\section{Modelos de previsión de la progresión del área de fisuras}

Las investigaciones iniciadas en la década de 1970 por la Empresa Brasilera de Planificación de Transportes (GEIPOT, 1981) resultaron en la Investigación del Interrelacionamiento de los Costos de Construcción, Conservación y Utilización de las Carreteras (PICR, Pesquisa do Interrelacionamento de Custos de Construção, Conservação e Utilização de Rodovias). En esa investigación, Queiroz (1981) utilizó datos obtenidos de la observación de 63 secciones de pavimento en servicio, de la malla carretera comprendida en el cuadrilátero Brasilia, Goiânia, Belo Horizonte y São Paulo.

El objetivo era desarrollar modelos para estimar el Coeficiente de Irregularidad (CI) y la evolución de las fisuras de los pavimentos (CR). Fue desarrollado un planeamiento factorial para la elección de secciones estudiadas, el análisis de regresión (Tabla 1) fue usado para la determinación de los parámetros del modelo estadístico y las variables independientes usadas fueron el Tráfico, la Edad (desde su construcción inicial o después de algún recapeamiento), las Deflexiones Recuperables y el Número Estructural corregido del pavimento. El modelo para la estimativa del CR desarrollado por Queiroz (1981) es mostrado en la Ecuación (1).

$$
C R=-18,53+0,04564 \log N+0,005011 B \times A \times \log N \quad \mathrm{R}^{2}=0,64
$$

En la que: $\quad A-$ edad del pavimento desde su construcción o recapeamiento, en años;

$B$ - deflexión en la viga Benkelman $(0,01 \mathrm{~mm})$;

$E R$ - variable indicadora del estado (0 como construido y 1 como recapeado);

$N$ - número equivalente de operaciones del eje padrón de $80 \mathrm{kN}$;

$T R$ - variable indicadora del tipo de revestimiento (0 para concreto asfáltico);

$S N C$ - número estructural corregido. 
Tabla 1 - Estadístico del análisis de regresión

\begin{tabular}{lccc}
\hline \multicolumn{1}{c}{ Parámetro } & Coeficiente & Error & $\mathrm{F}$ \\
\hline Coeficiente Lineal & $-18,530$ & - & - \\
$B \times \log N$ & 0,04564 & 0,01089 & 17,55 \\
$B \times A \times \log N$ & 0,005011 & 0,0007226 & 48,08 \\
\hline
\end{tabular}

Paterson (1987) desarrolló las ecuaciones de desempeño utilizadas en el programa computacional HDM-III (The highway design and maintenance starndards model, Watanada et al., 1987), herramienta utilizada en el planeamiento y programación de inversiones y de análisis de actividades de mantenimiento y rehabilitación de carreteras. Fue considerada la misma base empírica utilizada por Queiroz (1981), que incluía una gran cantidad de datos observados a lo largo del tiempo, tales como: irregularidad longitudinal, fisuras, desgaste, defectos superficiales, deformaciones permanentes, actividades de mantenimiento, tráfico y pluviometría.

El modelo para la estimativa de progresión de área de fisuras de la camada de revestimiento de concreto asfáltico desarrollado por Paterson (1987) está compuesto por las Ecuaciones (2) a (6). La curva desarrollada era una función del tipo $S$, no lineal.

$$
\begin{gathered}
\text { TYCRA }=K_{c i}\left(F_{c} \times R E L I C \times C R T\right) \\
R E L I C=4,21^{\left(0,14 \times S N C-17,1 \frac{Y E 4}{S N C}\right)} \\
\Delta A C R A_{d}=K_{c p} \times C R P \times Z_{a}\left[\left(Z_{a} \times a_{i} \times b_{i} \times \Delta T C R A+S C R A_{a}\right)^{b}\right]^{1 / b}-S C R A_{a} \\
C R P=1-0,12 C R T \\
\Delta T C R A=(A G E 2-T Y C R A) \operatorname{se}(A G E 2-1)<T Y C R A \leq A G E 2 \mathrm{e} \\
A C R A_{a}=0
\end{gathered}
$$

en donde: $\quad$ RELIC - pavimentos de concreto asfáltico sobre bases granulares;

$K_{c i}$ - factor de deterioración para inicio de la fisuración, calculado por la relación entre la edad esperada del revestimiento y la edad prevista por el modelo HDM - III, para el mismo volumen de tráfico;

YE4 - número de operaciones del eje equivalente de $80 \mathrm{kN}$ para el año de análisis, calculado por el método de la AASHTO (1986), en millones por carril de tráfico; 
$\triangle A C R A_{d}-$ mudanza prevista en el área de fisuras. Durante el año de análisis, debido al deterioro, en porcentaje del área total del revestimiento;

TYCRA - número promedio de años para el aparecimiento de fisuras;

$C R T$ - tiempo estimado de prórroga para el inicio de la fisuración debido a la realización de los servicios de mantenimiento, en años;

$K_{c p}$ - factor de deterioro para progresión de las fisuras;

$Z_{a}=1$, si $A C R A<50$ y $Z_{a}=-1 \geq 50$;

$a_{i}$ e $b_{i}$ - coeficientes determinados en función del tipo de revestimiento ( $a=$ 1,84 e $b=0,45$ para concreto asfáltico);

$\triangle T C R A=1$ se $T Y C R A \leq(A G E 2-1)$ o ACRA $_{a}>0 ;$

AGE2 - edad del pavimento desde la construcción, ejecución de camada sellante, rehabilitación o reconstrucción, en años;

$S C R A_{a}-$ mínimo $\left(A C R A_{a}, 100-A C R A_{a}\right)$;

$S C R A_{a}$ - máximo $\left(S C R A_{a} 0,5\right)$ si $A C R A_{a}>0,5$;

$A C R A_{a}$ - porcentaje de área de fisuras del pavimento igual a: $A C R A_{a}=0$, si $\triangle T C R A=0 ;: A C R A_{a}=0,5$ si $0<\triangle T C R A<1 ;: A C R A_{a}=A C R A$.

Marcon (1996) desarrolló modelos de previsión de desempeño con base en dados de la red carretera del Estado de Santa Catarina. Los datos, colectados en 1990, eran relacionados a deflexiones, irregularidades de superficie, defectos, materiales empleados, espesuras de las camadas y tráfico, correspondientes a tres regiones en que fue subdividido el Estado.

El desempeño de los pavimentos fue representado por las siguientes variables dependientes: coeficiente de irregularidad, deflexiones, Índice de Gravedad Global (IGG), fisuración total y ahuellamiento. La variable independiente fue el intervalo de tiempo, en años, o el número de operaciones del eje padrón de $80 \mathrm{kN}$. Fueron evaluadas cinco tipos de ecuaciones: lineal, logarítimica, polinomial, exponencial y potencial. El análisis de regresión mostró valores bajos de $\mathrm{R}^{2}$ entre las variables ATR y EDAD, Ecuación (7).

$$
A T R=1,5124 \times E D A D-6,8923 \quad R^{2}=0,32
$$

en donde: $\quad A T R$ - porcentaje del área del pavimento con fisuración total;

$E D A D$ - edad del pavimento desde su construcción en años. 


\section{Planificación factorial}

La planificación factorial es una herramienta estadística utilizada para investigar las diferencias de las respuestas provenientes de las variaciones en los factores, denominados de efectos principales, así como los efectos de interacción entre los factores. Inicialmente, son escogidas las variables independientes que se desean estudiar y se selecciona un número fijo de niveles para cada una de las variables, o factores, que son combinadas en una estructura experimental $l_{1} x l_{2} x \ldots l_{k}$, admitiéndose $l_{1}$ niveles para la primer variable, $l_{2}$ para la segunda, $\ldots$, y $l_{k}$ para la $k^{e ́ s i m a}$ (Box, Hunter y Hunter, 1978). En este trabajo la planificación factorial fue empleada con el objetivo de organizar los datos disponibles en el banco de datos de carreteras del DERBA, de tal manera que las matrices factoriales facilitasen el posterior análisis estadístico y la formulación del modelo de desempeño para pavimentos de concreto asfáltico caliente.

Para el desarrollo de las matrices factoriales fueron adoptados dos niveles para cada factor, de modo que fueron obtenidos experimentos factoriales del tipo $2^{3}=8$. No fue posible aumentar el número de niveles para 3 o más, ya que los intentos resultaron en celdas vacías en las matrices factoriales. También fueron previstas réplicas para las celdas de las matrices factoriales, pues son importantes para la estimativa del error experimental. Las réplicas son repeticiones de las evaluaciones en secciones del pavimento pertenecientes a una misma celda de la matriz factorial, cuyos resultados fueron obtenidos con los mismos equipamientos, procedimientos y espacio de inferencia.

Los niveles fueron escogidos de acuerdo con la distribución de los factores: edad del pavimento (ID) tráfico desde la abertura del pavimento hasta el año 2004 (TA) y pluviometría (PL). Los trechos estudiados por el DERBA (2005) hasta el año 2004 fueron 78. La Tabla 1 presenta, para esos 78 trechos, los valores promedio, mínimo y máximo para los factores ID, TA y PL. Como se puede notar en la Tabla 2, los valores promedio de los valores no coincidieron con los utilizados para la delimitación de los niveles de la planificación factorial, para las variables ID, TA y PL, entretanto, los valores fueron bastante aproximados. 
Tabla 2 - Valores promedios, mínimos y máximos de los factores ID, TA e PL, en los 78 trechos de pavimento de concreto asfáltico caliente

\begin{tabular}{lccc}
\hline Factor & Promedio & Mínimo & Máximo \\
\hline ID & 24 & 6 & 40 \\
TA & 2.013 .039 & 35.002 & 4.705 .610 \\
PL & 888 & 383 & 1934 \\
\hline
\end{tabular}

Los valores de las réplicas utilizadas para el desarrollo de los modelos fueron escogidos de manera aleatoria, para las celdas que presentaron más de dos valores. Los valores límites de los niveles de los factores fueron escogidas de manera que las celdas en la estructura del experimento factorial fuesen completadas con la cantidad de trechos aproximadamente igual. El planeamiento factorial para los trechos de pavimento estudiados es presentado a continuación.

Variable dependiente del área total de fisuras $\left(\mathrm{A}_{\mathrm{TF}}\right)$ :

Factor ID: edad del pavimento, con dos niveles, codificados por $\mathrm{i}_{1}(-1)$ e $\mathrm{i}_{2}(+1)$ :

- $\quad$ nivel id ${ }_{1}$ : edad del pavimento $\leq 26$ años;

- nivel $\mathrm{id}_{2}$ : edad del pavimento > 26 años.

Factor TA: tráfico desde su abertura hasta el año 2004, con dos niveles, codificados por $\mathrm{n}_{1}(-1)$ e $\mathrm{n}_{2}(+1)$ :

- nivel ta $1 \leq 2,48 \times 10^{6}$ tráfico operaciones del eje padrón de $80 \mathrm{kN}$;

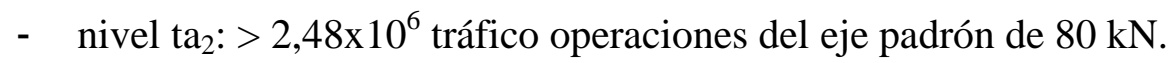

Factor PL: pluviometría, con dos niveles, codificados por $\mathrm{p}_{1}(-1)$ e $\mathrm{p}_{2}(+1)$ :

- $\quad$ nivel $\mathrm{pl}_{1}$ : pluviometría $\leq 720 \mathrm{~mm} / \mathrm{año}$

- nivel $\mathrm{pl}_{2}$ : pluviometría $>720 \mathrm{~mm} / \mathrm{año}$.

\section{Formulación del modelo de previsión de la progresión del área de fisuras}

\subsection{Análisis exploratorio de datos (AED)}

El AED es una filosofía de análisis de datos que emplea una variedad de técnicas, en su mayoría gráficas, para maximizar la percepción dentro de un grupo de datos, revelar las variables más importantes y obtener conclusiones sobre la significancia estadística de los factores (NIST/SEMATECH, 2008). La aplicación de los análisis gráficos se diferencia en el 
foco y en la sensibilidad, lo que puede mostrar diferentes aspectos con relación a los datos y, cuando técnicas múltiples presentan redundancia, existe mayor confianza en lo relacionado al alcance de las conclusiones.

Las técnicas gráficas escogidas para este trabajo fueron: el gráfico de dispersión, el gráfico de dispersión de los promedios, el gráfico de efectos y el gráfico de distribución de probabilidad semi-normal. Los valores utilizados en los gráficos fueron los promedios aritméticos de las réplicas de cada celda de la matriz. La Tabla 3 presenta la matriz factorial con los resultados de los levantamientos de $\mathrm{A}_{\mathrm{TF}}$ de los trechos utilizados para la realización del AED y el desarrollo del modelo.

Tabla 3 - Matriz factorial con los resultados de los levantamientos de ATF

\begin{tabular}{|c|c|c|c|c|}
\hline \multirow{3}{*}{ Edad (A) } & \multicolumn{4}{|c|}{ Tráfico Acumulado (B) } \\
\hline & \multicolumn{2}{|c|}{$\stackrel{\text { ta }_{1}}{\text { Pluviometría ( C ) }}$} & \multicolumn{2}{|c|}{$\begin{array}{c}\mathrm{ta}_{2} \\
\text { Pluviometría ( C ) }\end{array}$} \\
\hline & $\mathrm{pl}_{1}$ & $\mathrm{pl}_{2}$ & $\mathrm{pl}_{1}$ & $\mathrm{pl}_{2}$ \\
\hline \multirow{2}{*}{$\mathrm{id}_{1}$} & 16,50 & 27,78 & 57,00 & 38,75 \\
\hline & 8,75 & 9,29 & 25,39 & 35,00 \\
\hline Promedio aritmético & 12,63 & 18,54 & 41,20 & 36,80 \\
\hline \multirow{2}{*}{$\mathrm{id}_{2}$} & 63,75 & 20,00 & 65,00 & 85,00 \\
\hline & 55,00 & 5,00 & 58,00 & 71,00 \\
\hline Promedio aritmético & 59,40 & 12,50 & 61,50 & 78,00 \\
\hline
\end{tabular}

El gráfico de dispersión (Figura 1) auxilia en la determinación de los factores más importantes, que son aquellos que conducen a una variación significativa en la dispersión de los valores de la variable respuesta, cuando se pasa del nivel inferior ( - ) para el nivel superior $(+)$, al compararse con el valor de su promedio global (línea punteada). En el eje vertical aparecen los valores promedio de las réplicas para la variable respuesta, para cada celda de la matriz factorial y para cada uno de los factores, obtenidos en la Tabla 3.

En el eje horizontal son presentados los factores con sus dos niveles $(-\mathrm{y}+)$, conforme la secuencia presentada en la leyenda de la Figura 1. Por ejemplo, los valores de la $A_{T F}$ para el nivel inferior ( - ) del factor edad del pavimento, representado en el gráfico por rombos sin relleno, son los valores: $12,63,18,54,41,20$ y 36,80. Los valores de $A_{\mathrm{TF}}$ para el nivel superior $(+)$, representados por rombos con relleno, son los valores 59,40, 12,50, 61,50 y 78,00. El mismo proceso es repetido con los valores promedio de las réplicas para los niveles de los factores: tráfico y pluviometría. Se trata de un análisis primario de los datos para determinar 
cómo la variable respuesta se relaciona con los factores y cómo los datos cambian en función de los diferentes niveles de un factor.

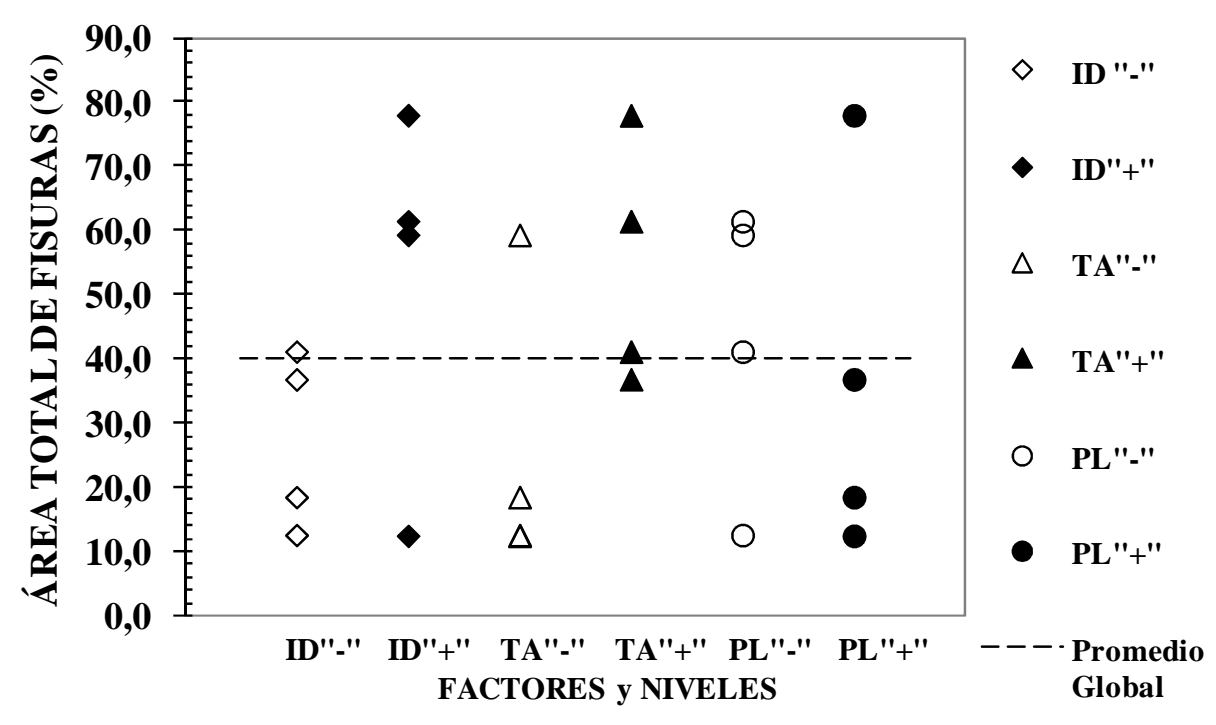

Figura 1 - Gráfico de dispersión de los valores de área de fisuras

En el gráfico de dispersión de promedios (Figura 2), un determinado factor es importante si presenta una variación significativa en la dispersión de los valores promedio de la variable respuesta, cuando se pasa del nivel inferior ( - ) para el nivel superior $(+)$, en comparación con el valor de su promedio global.

En el eje vertical aparecen los valores promedio para la variable respuesta, para un nivel dado y para cada uno de los factores, obtenidos en la Tabla 3, mientras que en el eje horizontal son presentados los factores con sus dos niveles $(-\mathrm{y}+)$, conforme la secuencia presentada en la leyenda de la Figura 2. Por ejemplo, el valor de la $A_{T F}$ para el nivel inferior ( - ) del factor edad del pavimento, presentado en el gráfico con rombos sin relleno, es el promedio de los valores $12,63,18,56,41,20$ y 36,80 . Para el nivel superior $(+)$ del $A_{T F}$, en rombos con relleno, es el promedio de los valores 59,40, 12,50, 61,50 y 78,00. 


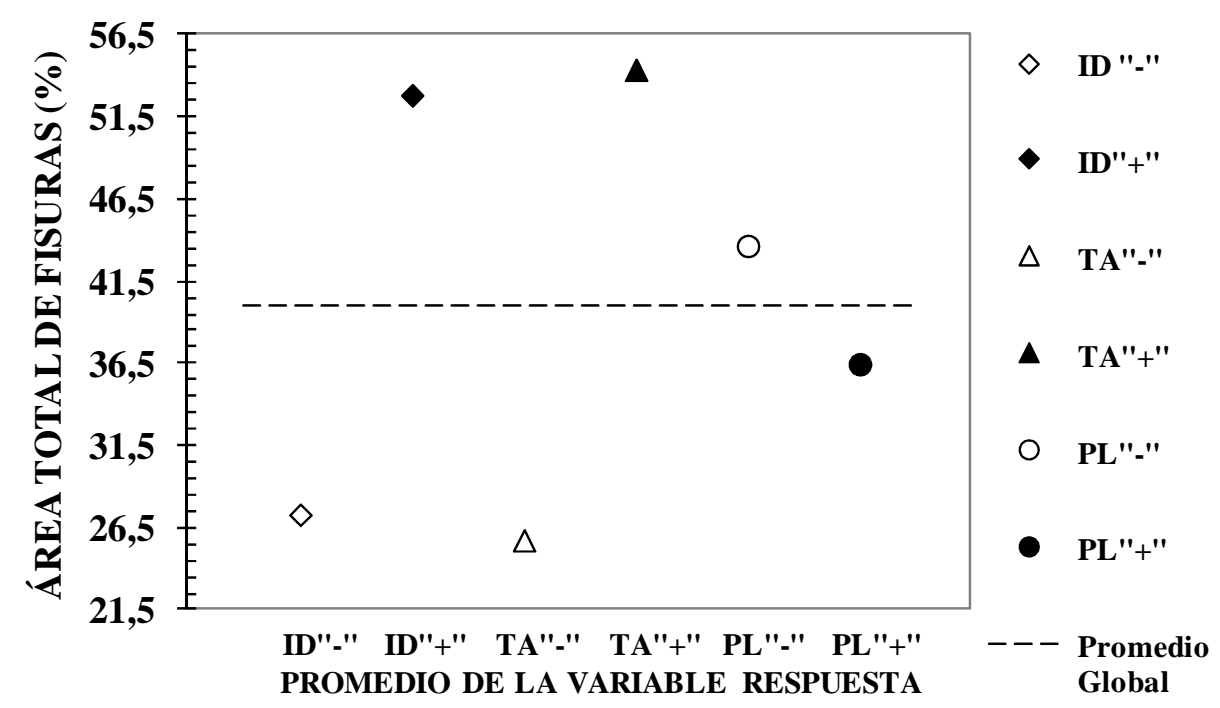

Figura 2 - Gráfico de dispersión de los valores promedio de los factores

El gráfico de efectos (Figura 3) ayuda en el agrupamiento de los factores y sus interacciones en dos categorías: importantes y no importantes. En el eje vertical aparecen los valores absolutos de los efectos estimados para los factores y sus interacciones y en el eje horizontal es mostrada la secuencia de los factores y sus interacciones, del más importante al menos importante. Para experimentos factoriales $2^{\mathrm{k}}$, la estimativa de los efectos $(E)$ utiliza el método de mínimos cuadrados, según la Ecuación (8):

$$
E=\bar{Y}(+)-\bar{Y}(-)
$$

en donde: $\quad \bar{Y}(+)$ : valor promedio de la variable respuesta para el nivel superior $(+)$;

$\bar{Y}(-)$ : valor promedio de la variable respuesta para el nivel inferior (-).

Esa es una simplificación válida debido a la ortogonalidad que ocurre en los experimentos de dos niveles. Si un factor o la interacción entre factores es importante, el promedio del nivel (+) del factor será considerablemente diferente del promedio de su nivel (-), y el valor absoluto de la diferencia será mayor, cuando es comparado con los demás. Por otro lado, factores o interacciones sin importancia tendrán pequeñas diferencias en los promedios y, así, el valor absoluto será pequeño. La Tabla 4 presenta el resumen del cálculo de los efectos estimados de los factores e interacciones sobre la variable dependiente Irregularidad Longitudinal. 
Tabla 4 - Resumen del cálculo de los efectos estimados de los factores e interacciones

\begin{tabular}{lccccccc}
\hline & $\mathbf{1}$ & $\mathbf{2}$ & $\mathbf{3}$ & $\mathbf{1 2}$ & $\mathbf{1 3}$ & $\mathbf{2 3}$ & $\mathbf{1 2 3}$ \\
\hline & ID & TA & PL & ID*TA & ID*PL & TA*PL & ID*TA*PL \\
Y $(+)$ & 52,844 & 54,392 & 36,477 & 42,664 & 36,079 & 46,719 & 49,275 \\
Y $(-)$ & 27,306 & 25,758 & 43,673 & 37,486 & 44,071 & 33,431 & 30,875 \\
E & 25,538 & 28,634 & $-7,196$ & 5,179 & $-7,991$ & 13,288 & 18,400 \\
\hline
\end{tabular}

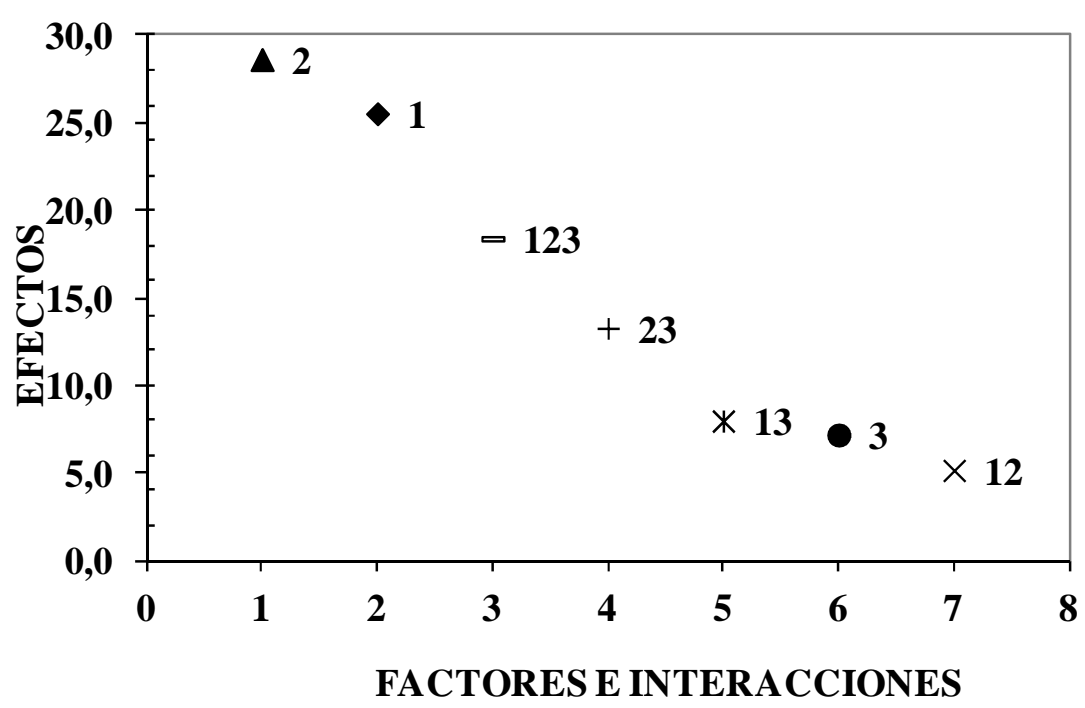

Figura 3 - Gráfico de efectos de los factores e interacciones

El gráfico de distribución de probabilidad semi-normal (Figura 4) muestra los efectos contra los valores esperados de su distribución normal, sin embargo, apenas los valores positivos de la curva de distribución normal son considerados. Consecuentemente, solamente valores normales positivos son indicados en el eje horizontal. Los efectos no importantes tienden a localizarse fuera de esta distribución centrada en cero. Para hacer distinción entre factores importantes y no importantes es necesario identificar dónde hay aglomeración de los efectos de los factores y sus interacciones, en línea, próximo de cero. 


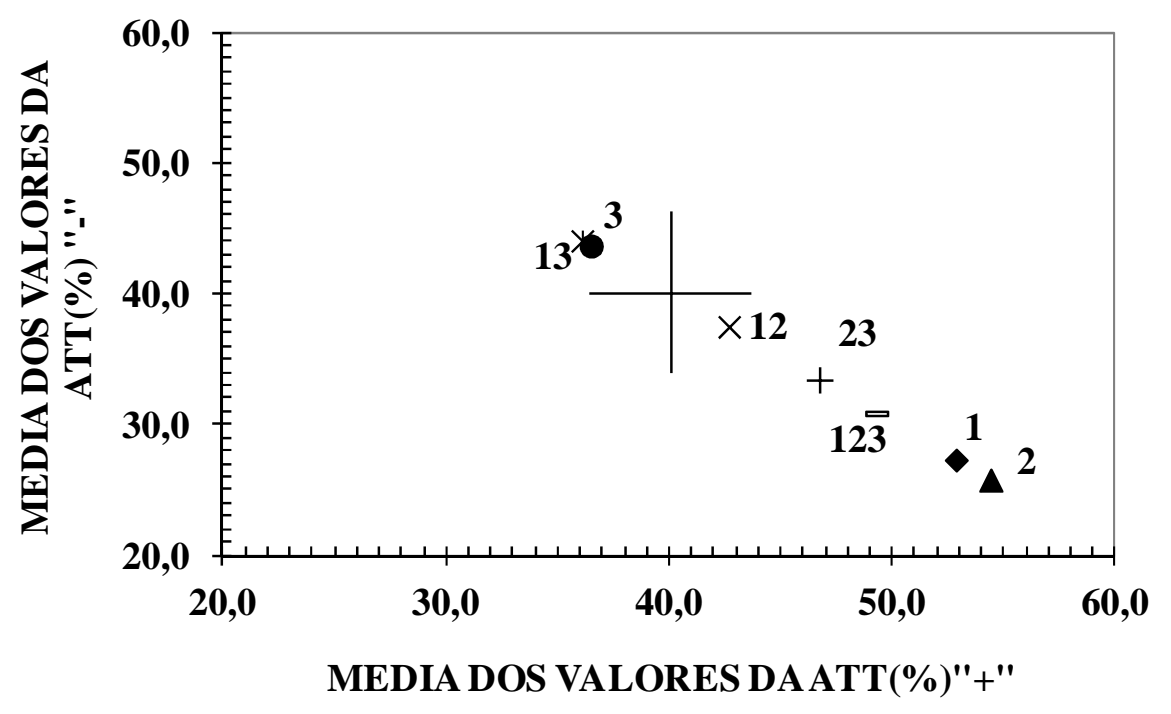

\section{Figura 4 - Gráfico de distribución de probabilidad semi-normal} de los factores e interacciones

A partir del análisis de los gráficos mostrados en las Figuras 1 a 4, se obtuvo una jerarquización en orden decreciente de la importancia de los factores y de las interacciones: tráfico, edad del pavimento, interacción entre los factores edad del pavimento, tráfico acumulado y pluviometría, interacción entre los factores tráfico acumulado y pluviometría. Las demás interacciones y la Pluviometría fueron consideradas no importantes.

\subsection{Análisis de varianza}

El análisis de varianza es una herramienta estadística que tiene por objetivo verificar si existe una diferencia significativa entre los promedios provenientes de muestras distintas y si los factores ejercen influencia en el modelo. La distribución $F$ es utilizada por el ANOVA en la decisión de aceptar o rechazar la hipótesis de igualdad entre los grupos de datos (hipótesis nula). La Tabla 5 presenta el resumen del cálculo del ANOVA de los factores y de sus interacciones. La variable dependiente considerada es la irregularidad longitudinal, para un nivel de significancia de $5 \%\left(\alpha=0,05, F_{\text {crítico }}=5,32\right)$. 
Tabla 5 - Resumen del ANOVA para el experimento factorial

\begin{tabular}{lccccccc}
\hline Factor & $\begin{array}{c}\text { Suma } \\
\text { Cuadrática }\end{array}$ & $\begin{array}{c}\text { Grados } \\
\text { Libertad }\end{array}$ & $\begin{array}{c}\text { Promedio } \\
\text { Cuadrático }\end{array}$ & Coeficiente & $F_{o}$ & Significativo & p-valor \\
\hline ID & 2608,681 & 1 & 2608,681 & 12,769 & 21,272 & SI & 0,001728 \\
TA & 3279,595 & 1 & 3279,595 & 14,317 & 26,743 & SI & 0,000852 \\
PL & 207,151 & 1 & 207,151 & $-3,598$ & 1,689 & NO & 0,229907 \\
ID*TA & 107,273 & 1 & 107,273 & 2,589 & 0,875 & NO & 0,377016 \\
ID*PL & 255,432 & 1 & 255,432 & $-3,996$ & 2,083 & NO & 0,186948 \\
TA*PL & 706,244 & 1 & 706,244 & 6,644 & 5,759 & SI & 0,043192 \\
ID*TA*PL & 1354,222 & 1 & 1354,222 & 9,200 & 11,043 & SI & 0,010492 \\
Error & 981,075 & 8 & 122,634 & & & & \\
Total & 9499,673 & 15 & & 40,075 & & & 0,000001 \\
\hline
\end{tabular}

El análisis de regresión fue usado para la determinación de los parámetros del modelo estadístico para la previsión del $\mathrm{A}_{\mathrm{TF}}$, conforme presentado en la Ecuación (9) $\left(\mathrm{R}^{2}=0,83\right)$. Las ecuaciones polinomiales correspondientes a los factores Edad del Pavimento, Tráfico y Pluviometría son presentadas en las Ecuaciones (10), (11) y (12).

$$
\begin{gathered}
A_{T F}=40,1+12,7 P(I D)+14,3 P(T A)+6,6 P(T A) P(P L)+9,2 P(I D) P(T A) P(P L) \\
P(I D)=\frac{I D-26}{13} \\
P(T A)=\frac{T A-2,4 \times 10^{6}}{3,43 \times 10^{6}} \\
P(P L)=\frac{P L-965}{842}
\end{gathered}
$$

en donde: $\quad P(I D)$ : ecuación polinomial para el factor edad desde la construcción;

$P(T A)$ : ecuación polinomial para el factor tráfico;

$P(P L)$ : ecuación polinomial para el factor pluviometría;

ID: valor cuantitativo de la variable Edad del Pavimento, en años;

$T A$ : valor cuantitativo de la variable tráfico, en número de aplicaciones equivalentes del eje padrón;

$P L$ : valor cuantitativo de la variable pluviometría, en $\mathrm{mm}$ anuales. 
Para la verificación de la adecuación del modelo estadístico se utilizó la recta de ajuste de los datos previstos y los observados, con determinación del coeficiente de correlación (r) correspondiente (Figura 5), el análisis de residuos (Figura 6) y el gráfico de probabilidad normal de los residuos (Figura 7). Debe destacarse que el conjunto de datos observados, utilizados para la validación del modelo, también fue colectado en la etapa del levantamiento en campo, en secciones de pavimento diferentes de las que fueron consideradas para el desarrollo del modelo de previsión de desempeño de la $\mathrm{A}_{\mathrm{TF}}$.

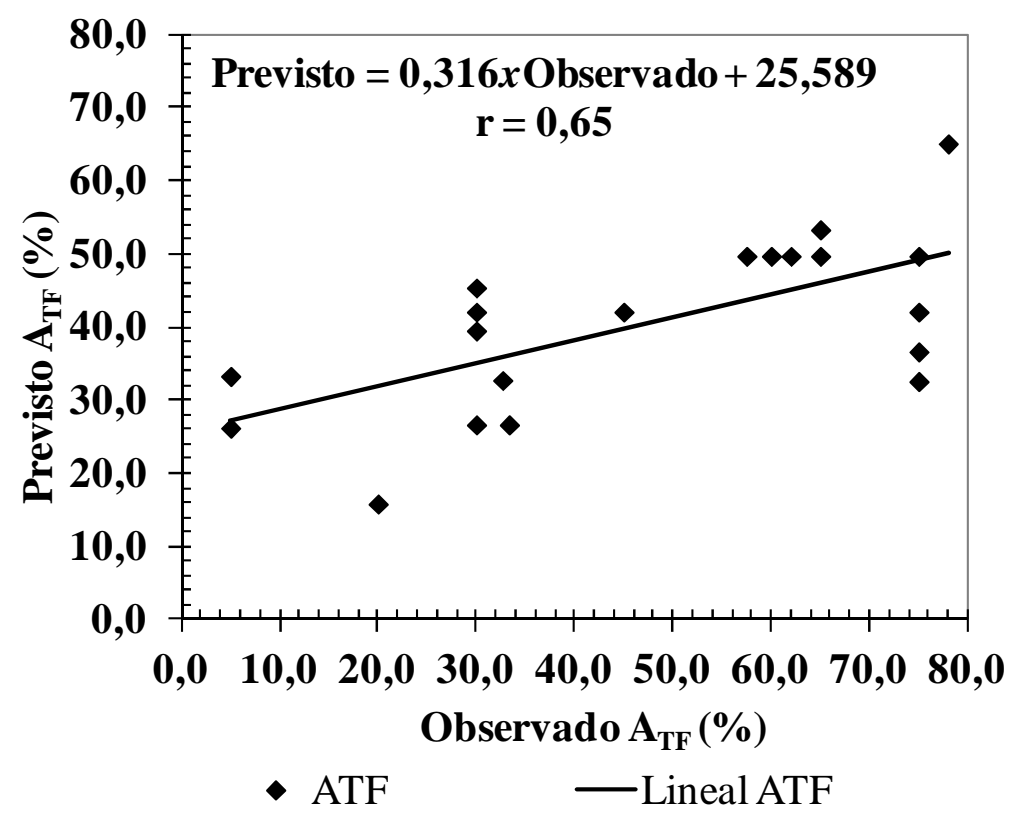

Figura 5 - Correlación de los valores observados del área total de fisuras con los valores previstos por la ecuación (9)

Por el análisis de la Figura 5 puede notarse que la recta de regresión lineal de la $\mathrm{A}_{\mathrm{TF}}$ presentó un ajuste al conjunto de pares de puntos observados y previstos, con $r=0,65$. La Figura 6 muestra que existe una distribución aleatoria en torno de cero, lo que indica la igualdad entre las varianzas, mientras que la Figura 7 indica la normalidad de los datos, pues los residuos están distribuidos aproximadamente a lo largo de una línea recta. 


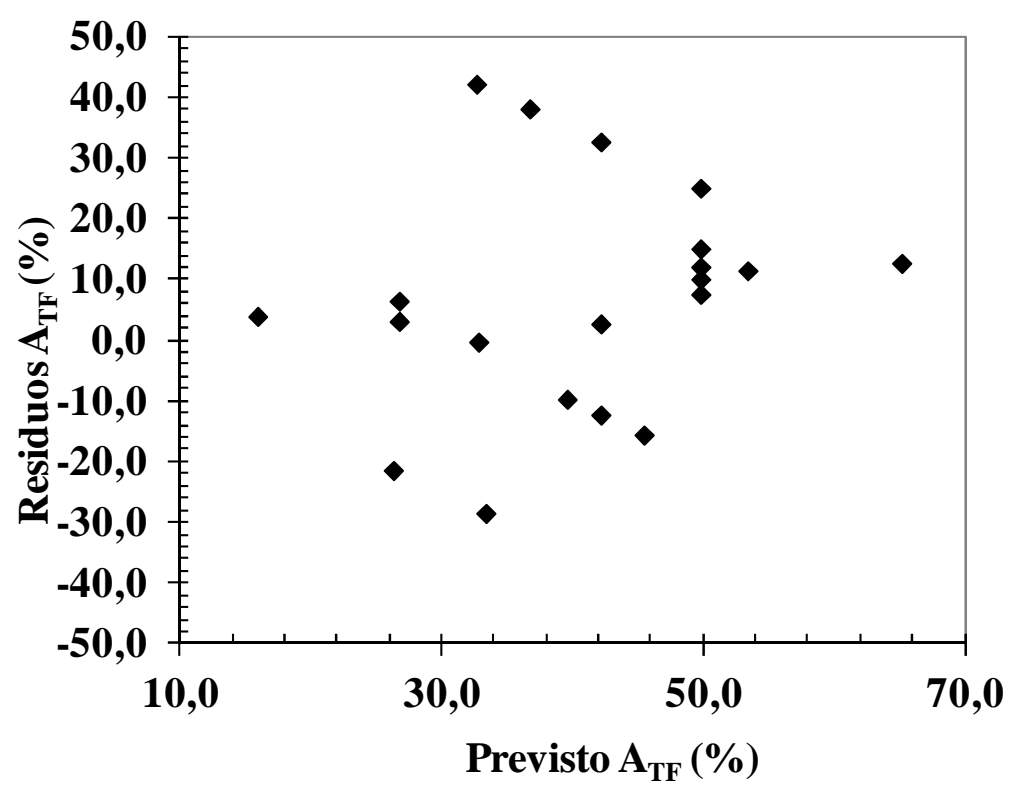

Figura 6 - Gráfico de residuos del modelo de desempeño del área total de fisuras obtenido

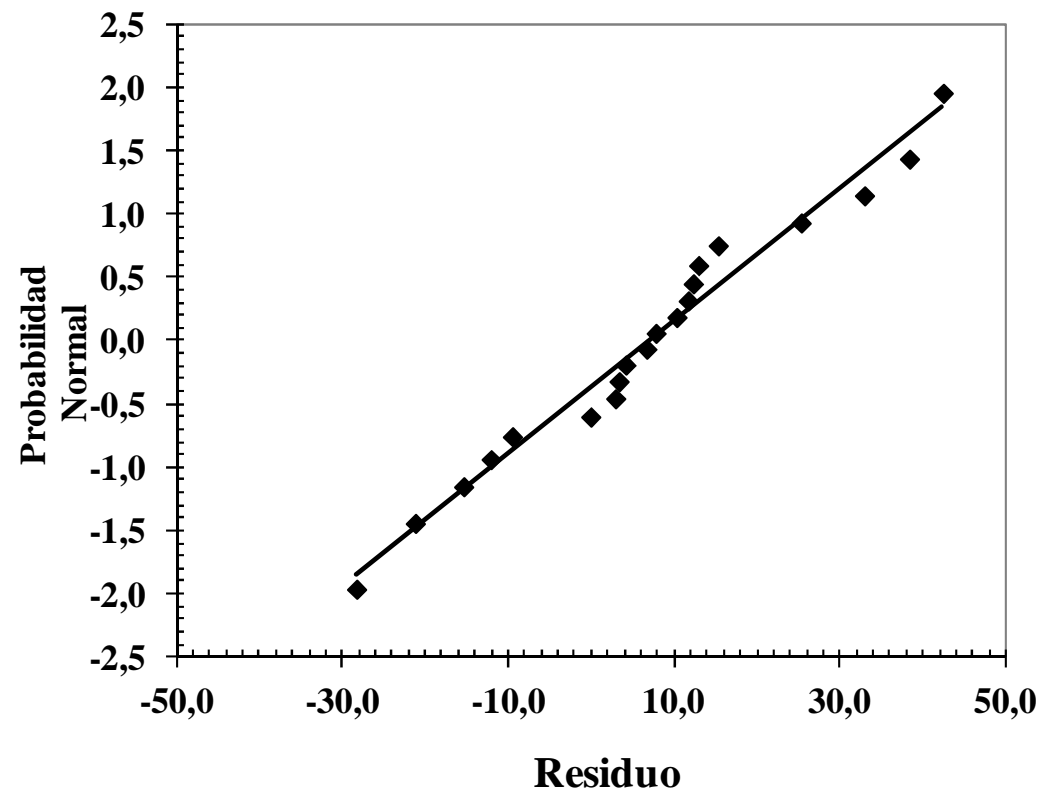

Figura 7 - Gráfico de distribución de probabilidad normal de los residuos

La Tabla 6 presenta los valores observados, los previstos y los residuos de la variable dependiente área total de fisuras (\%), para los trechos que no fueron utilizados en el desarrollo del modelo. Los valores previstos fueron calculados a través de la Ecuación (9). 
Tabla 6 - Valores del área total de fisuras observados y previstos por la ecuación (9)

\begin{tabular}{lccc}
\hline Codigo del DERBA & $\begin{array}{c}\mathrm{A}_{\mathrm{TF}}(\%) \\
\text { (observado) }\end{array}$ & $\begin{array}{c}\mathrm{A}_{\mathrm{TF}}(\%) \\
\text { (previsto) }\end{array}$ & Residuos \\
\hline 001EBA0185 & 5,00 & 26,36 & $-21,36$ \\
120EBA0125 & 75,00 & 32,72 & 42,28 \\
130EBA0235 & 30,00 & 39,65 & $-9,65$ \\
220EBA0065 & 30,00 & 45,52 & $-15,52$ \\
233EBA0095 & 20,00 & 15,98 & 4,02 \\
262EBA0125 & 45,00 & 42,22 & 2,78 \\
262EBA0135 & 30,00 & 42,22 & $-12,22$ \\
262EBA0135 & 75,00 & 42,22 & 32,78 \\
270EBA0025 & 33,33 & 26,80 & 6,53 \\
270EBA0030 & 30,00 & 26,80 & 3,20 \\
274EBA0005 & 5,00 & 33,44 & $-28,44$ \\
275EBA0025 & 32,67 & 32,88 & $-0,21$ \\
499EBA0020 & 75,00 & 36,79 & 38,21 \\
512EBA0015 & 78,00 & 65,22 & 12,78 \\
535EBA0005 & 57,50 & 49,85 & 7,65 \\
535EBA0020 & 65,00 & 49,85 & 15,15 \\
AREMBEPE & 75,00 & 49,85 & 25,15 \\
Praia do Forte & 60,00 & 49,85 & 10,15 \\
P. de Sauipe & 62,00 & 49,85 & 12,15 \\
São F.do Conde & 65,00 & 53,45 & 11,55 \\
\hline
\end{tabular}

\section{Análisis de los efectos de los factores}

La Tabla 7 presenta un resumen comparativo entre los resultados obtenidos por el AED y por el ANOVA. Puede notarse que los resultados del AED corroboran los resultados encontrados a través del ANOVA, tanto en relación a los efectos a los factores como a las interacciones. Los efectos de la edad del pavimento y del tráfico fueron significativos e importantes para el modelo, así como lo fue la interacción entre el tráfico acumulado y la pluviometría y la interacción entre los tres factores. 
Tabla 7 - Resumen comparativo entre los resultados del AED y del ANOVA

\begin{tabular}{lcccc}
\hline & \multicolumn{3}{c}{$\mathrm{A}_{\mathrm{TF}}$} \\
\cline { 2 - 5 } Factores & \multicolumn{2}{c}{ ANOVA } & \multicolumn{2}{c}{ AED } \\
& $\mathrm{F}_{0}$ & SI $/$ NO & E & SI/NO \\
& 21,27 & SI & 25,54 & SI \\
ID & 26,74 & SI & 28,63 & SI \\
TA & 1,69 & NO & $-7,20$ & NO \\
PL & 0,88 & NO & 5,18 & NO \\
ID*TA & 2,08 & NO & $-7,99$ & NO \\
ID*PL & 5,76 & SI & 13,29 & SI \\
TA*PL & 11,04 & SI & 18,40 & SI \\
ID*TA*PL & $11,0 y n c i a$ \\
\hline
\end{tabular}

Los efectos de los factores también fueron evaluados en gráficos de tridimensionales, pues la consideración de dos factores implica en un tercer factor constante o igual a cero. De esa forma es posible evaluar el comportamiento de dos variables, de forma simultánea. Las Figuras 8, 9 y 10 muestran los efectos de los factores edad, tráfico y pluviometría sobre el área total de fisuras $\left(\mathrm{A}_{\mathrm{TF}}\right)$, con base en el modelo de la ecuación (9). La Figura 8 muestra que el $A_{T F}$ aumenta cuando los factores edad y tráfico cambian del nivel inferior (-1) para el nivel superior (+1), siendo mayor el efecto del tráfico que el efecto de la edad del pavimento.

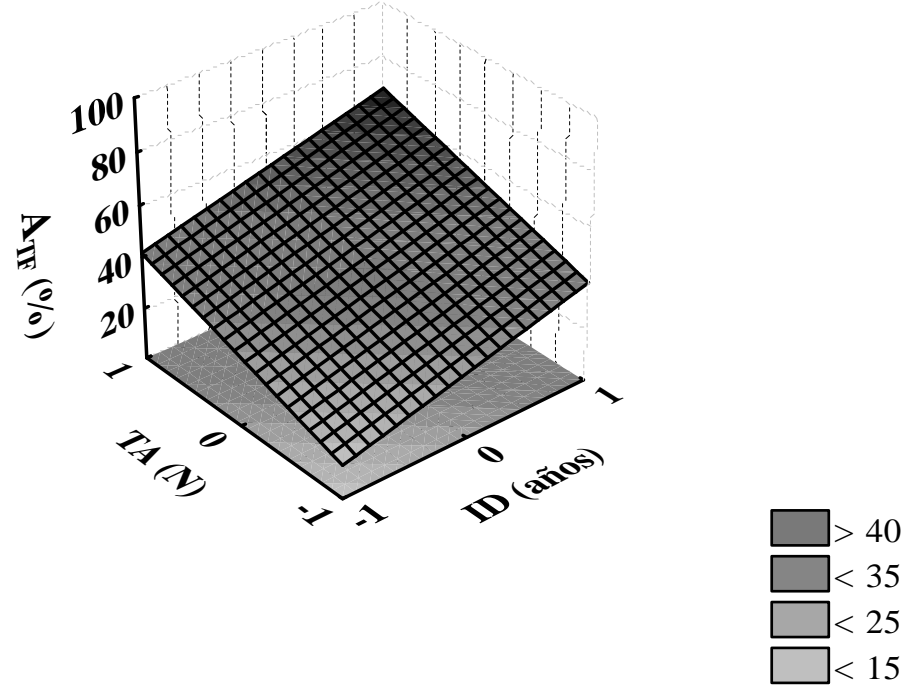

Figura 8 - Efectos de los factores edad y tráfico sobre el $\mathrm{A}_{\mathrm{TF}}$ 


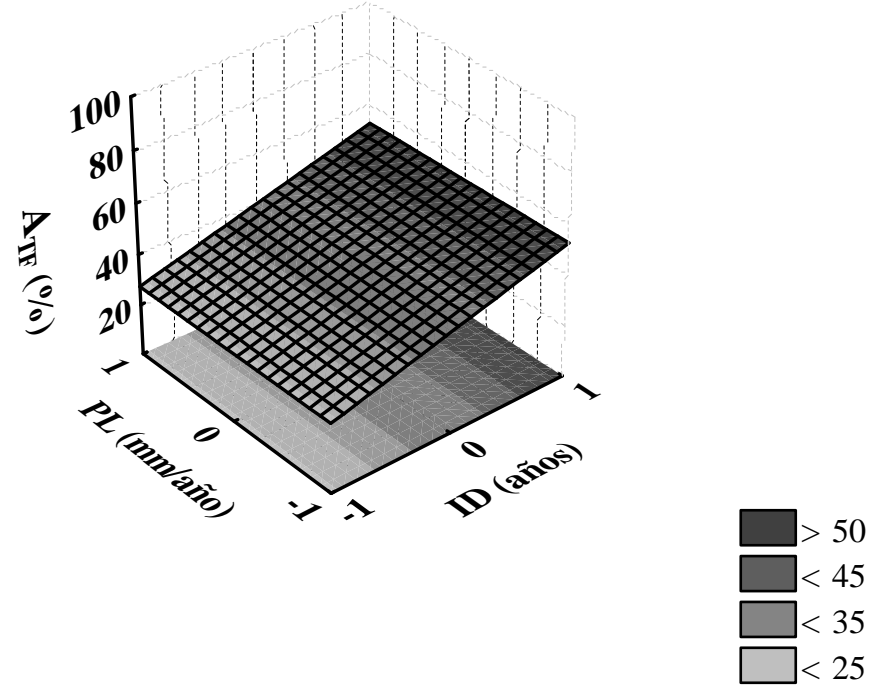

Figura 9 - Efectos de los factores edad y pluviometría sobre el $\mathrm{A}_{\mathrm{TF}}$

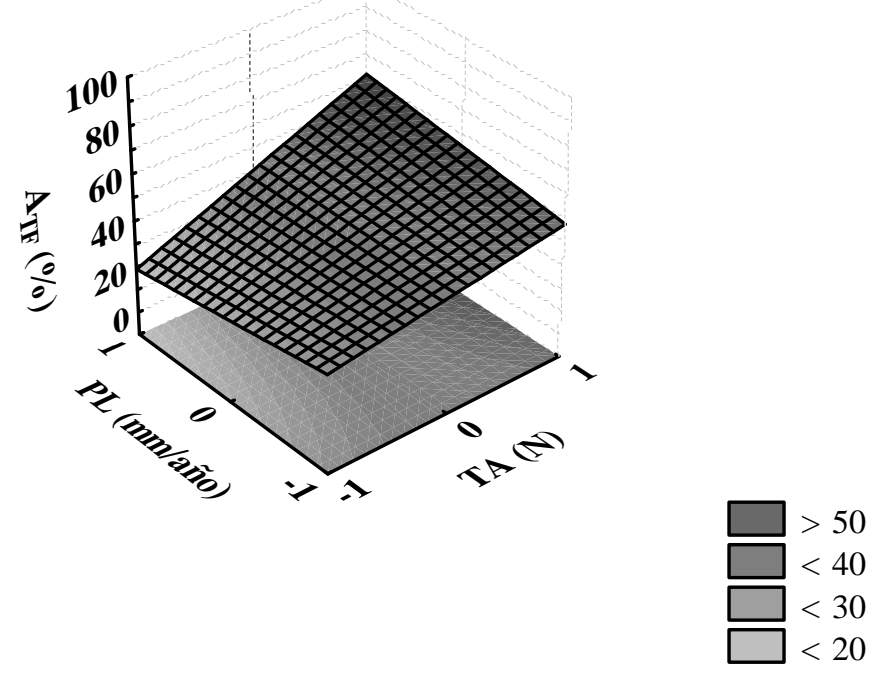

Figura 10 - Efectos de los factores tráfico y pluviometría sobre el $\mathbf{A}_{\mathrm{TF}}$

La Figura 9 muestra que el $\mathrm{A}_{\mathrm{TF}}$ aumenta cuando la edad cambia del nivel inferior (-1) para el nivel superior $(+1)$. Entretanto, el $\mathrm{A}_{\mathrm{TF}}$ se mantiene constante con la variación de la pluviometría, lo que significa que el efecto principal de la pluviometría no tiene efecto sobre el modelo. La Figura 10 muestra que el $\mathrm{A}_{\mathrm{TF}}$ aumenta cuando el tráfico cambia del nivel inferior (-1) para el nivel superior (+1). A pesar de no haber influencia del efecto principal de la pluviometría en el modelo, la variación en la ordenada indica el efecto de la pluviometría en la interacción TA*PL. 


\section{Análisis comparativo con otros modelos de desempeño}

El modelo de previsión del $\mathrm{A}_{\mathrm{TF}}$ desarrollado en este trabajo fue comparado con el modelo propuesto por Marcon (1996). El modelo de Paterson (1987) no presentó un buen ajuste con relación a los datos observados, principalmente para valores de edad del pavimento mayores a 15 años y valores de número equivalente de operaciones del eje padrón de $80 \mathrm{kN}$ superiores a $1 \times 10^{5}$. No fue posible la aplicación del modelo de Queiroz (1981), ya que no había información sobre las deflexiones recuperables de los trechos del banco de datos de carreteras del DERBA.

Los resultados previstos, calculados con la Ecuación (9), fueron comparados con los resultados obtenidos de la Ecuación (7), desarrollada por Marcon (1996). Los resultados son presentados en la Tabla 8. Los valores del área total de fisuras fueron calculados en función de la edad de los pavimentos.

Tabla 8 - Valores observados y los previstos por los modelos de desempeño de $A_{\text {TF }} y$ de Marcon (1996)

\begin{tabular}{ccccccc}
\hline \multirow{2}{*}{$\begin{array}{c}\text { Código del } \\
\text { DERBA }\end{array}$} & $\begin{array}{c}\mathrm{A}_{\mathrm{TF}}(\%) \\
\text { (observado) }\end{array}$ & $\begin{array}{c}\text { Edad } \\
\text { (años) }\end{array}$ & $\begin{array}{c}\text { Tráfico } \\
(\mathrm{N})\end{array}$ & $\begin{array}{c}\text { Pluviometría } \\
(\mathrm{mm} / \text { año })\end{array}$ & \multicolumn{2}{c}{$\mathrm{A}_{\mathrm{TF}}(\%)$ (previsto) } \\
\hline 001EBA0185 & & & & & & Marcon \\
120EBA0125 & 5,00 & 21,00 & $7,04 \mathrm{E}+05$ & 1025 & 26,36 & 24,87 \\
130EBA0235 & 75,00 & 27,00 & $2,77 \mathrm{E}+05$ & 686 & 32,72 & 33,94 \\
220EBA0065 & 30,00 & 23,00 & $3,62 \mathrm{E}+06$ & 508 & 39,65 & 27,89 \\
233EBA0095 & 30,00 & 36,00 & $4,82 \mathrm{E}+05$ & 585 & 45,52 & 47,55 \\
262EBA0125 & 20,00 & 10,00 & $1,23 \mathrm{E}+06$ & 686 & 15,98 & 8,23 \\
262EBA0135 & 45,00 & 25,00 & $3,87 \mathrm{E}+06$ & 496 & 42,22 & 30,92 \\
262EBA0135 & 30,00 & 25,00 & $3,87 \mathrm{E}+06$ & 496 & 42,22 & 30,92 \\
270EBA0025 & 75,00 & 25,00 & $3,87 \mathrm{E}+06$ & 496 & 42,22 & 30,92 \\
270EBA0030 & 33,33 & 22,00 & $7,22 \mathrm{E}+05$ & 1377 & 26,80 & 26,38 \\
274EBA0005 & 30,00 & 22,00 & $7,22 \mathrm{E}+05$ & 1377 & 26,80 & 26,38 \\
275EBA0025 & 5,00 & 29,00 & $8,28 \mathrm{E}+05$ & 1377 & 33,44 & 36,97 \\
499EBA0020 & 32,67 & 29,00 & $2,61 \mathrm{E}+05$ & 1025 & 32,88 & 36,97 \\
512EBA0015 & 75,00 & 29,00 & $7,75 \mathrm{E}+05$ & 686 & 36,79 & 36,97 \\
535EBA0005 & 78,00 & 40,00 & $4,71 \mathrm{E}+06$ & 1184 & 65,22 & 53,60 \\
535EBA0020 & 57,50 & 29,00 & $4,17 \mathrm{E}+06$ & 1184 & 49,85 & 36,97 \\
AREMBEPE & 65,00 & 29,00 & $4,17 \mathrm{E}+06$ & 1184 & 49,85 & 36,97 \\
Praia do Forte & 75,00 & 29,00 & $4,17 \mathrm{E}+06$ & 1184 & 49,85 & 36,97 \\
P. de sauipe & 60,00 & 29,00 & $4,17 \mathrm{E}+06$ & 1184 & 49,85 & 36,97 \\
São F do Conde & 62,00 & 29,00 & $4,17 \mathrm{E}+06$ & 1184 & 49,85 & 36,97 \\
\hline
\end{tabular}


Las rectas de ajuste para los modelos son presentadas en la Figura 11. En esa figura puede observarse que las inclinaciones de las rectas del modelo para previsión de $\mathrm{A}_{\mathrm{TF}} \mathrm{y}$ del modelo desarrollado por Marcon (1996), que considera solamente el factor edad, son aproximadamente iguales, lo que indica una tendencia de evolución del área total de fisuras semejante a lo largo del tiempo. También es posible observar un mejor resultado del modelo desarrollado en este trabajo, debido al mejor ajuste con relación a los datos observados y a la mejor correlación $(r=0,65)$.

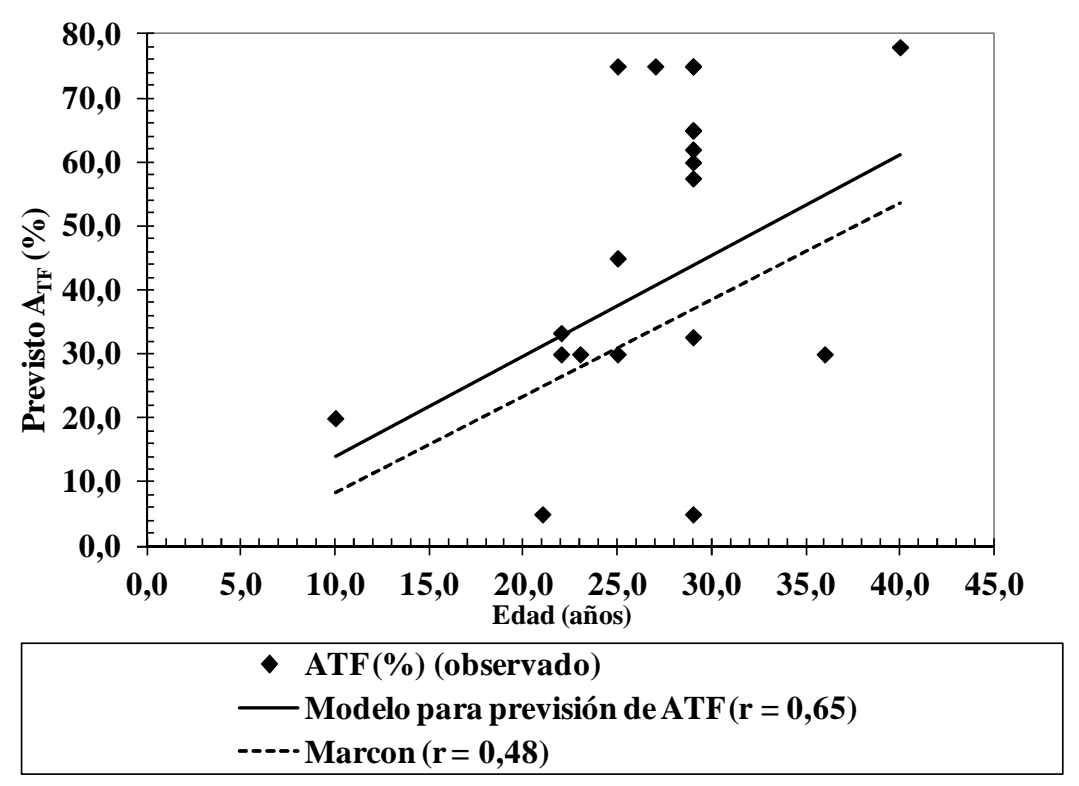

Figura 11 - Análisis comparativo del modelo de $A_{T F}$ con el modelo de Marcon (1996)

\section{Conclusiones}

En este trabajo fue desarrollado un modelo de desempeño para la previsión del área total de fisuras considerando los datos de la malla carretera pavimentada en concreto asfáltico caliente del Estado de Bahia, Brasil. Fueron utilizadas dos formas de análisis estadístico de los datos para evaluar la significancia de los factores y definir los parámetros de los modelos de desempeño, AED y ANOVA. La primera herramienta, se mostró simple y eficaz en la determinación de los efectos de los factores y sus interacciones y sus resultados fueron corroborados por los resultados obtenidos a través del análisis de varianza, tanto en lo relacionado a los efectos como a las interacciones. 
El modelo aquí desarrollado presentó un coeficiente de determinación $\left(\mathrm{R}^{2}=0,83\right)$ y mejor ajuste a los datos observados. En comparación con los modelos de Marcon (1986) y Paterson (1987), presentó mejor correlación entre los valores observados y los previstos, lo que demuestra evidencia del la validez de su utilización en análisis de redes carreteras constituidas por revestimientos asfálticos. Sin embargo, para conseguir mejores resultados para predecir la progresión del área de fisuras em pavimentos asfálticos en el estado de Bahía y otras redes de carreteras, es necesario desarrollar nuevos estudios para alimentar la base de datos, de manera que podamos mejorar la precisión y la exactitud del modelo desarrollado.

Vale resaltar que son necesarios estudios complementares que incluyan factores que no pudieron ser considerados en este trabajo, como la capacidad estructural del pavimento. El método aquí utilizado puede ser empleado también para esos casos, ya que se mostró capaz de definir las cantidades de datos colectados para la realización del experimento en campo, con reducción de costos y sin comprometer la precisión del modelo de desempeño obtenido.

También es sugerido comparar los modelos de desempeño desarrollados en este trabajo con los modelos de deterioro del HDM-4. Además, datos de otras regiones y mallas carreteras podrían ser utilizados para verificar la aplicabilidad del modelo de $\mathrm{A}_{\mathrm{TF}}$ aquí desarrollado.

\section{Agradecimientos}

Al CNPq por la concesión de una bolsa de doctorado, al Programa de Pos-Graduación en Ingeniería de Transportes de la EESC-USP, a la UFBA por el apoyo en los levantamientos de campo y al DERBA por ceder los datos para la ejecución de este trabajo. 


\section{Referências}

American Association of State Highway Transportation Officials - AASHTO (1986) Guide for design of pavement structures. Washington, DC.

Box, G. E. P., Hunter, W. G. and Hunter, J. S. (1978) Statistics for experimenters: an introduction to design, data analysis, and model building. EUA: John Wiley \& Sons.

Carey, W. N. and Irick, P. E. The Pavement Serviceability - Performance Concepts. Highway Research Bulletin 250, 1960.

Departamento de Infraestrutura de Transportes da Bahia - DERBA (2005) Sugestões Técnicas para Implementação do Programa de Restauração e Manutenção Rodoviária. Vol. 1 a 5. Salvador, BA.

Empresa Brasileira de Planejamento de Transportes - GEIPOT (1981) Pesquisa Sobre o Interrelacionamento dos Custos de Construção, Conservação e Utilização de Rodovias. Ministério dos Transportes, Brasília, DF.

Hass, R. and Hudson, W. R. (1978) Pavement management systems. United States of America: McGraw-Hill Book Company, 457 p.

Lytton, R. L. (1987) Concepts of Pavement Performance Prediction and Modeling. Proc., 2nd North American Conference on Managing Pavements, Toronto, Ontário, Canadá, 1987.

Marcon, F. M. (1996) Contribuição ao Desenvolvimento de um Sistema de Gerência de Pavimentos para a Malha Rodoviária Estadual de Santa Catarina. Tese (Doutorado), Centro Técnico aeroespacial, Instituto Tecnológico de Aeronáutica, São Paulo.

NIST/SEMATEC (2008) E-handbook of statistical methods. Disponible en: www.itl.nist.gov.

Paterson, W. D. O. (1987) Road Deterioration and Maintenance Effects: Models for Planning and Management. Published for the Word Bank, Washington, D. C.

Queiroz, C. A. V (1981) Performance prediction models for pavement management in Brazil. Thesis (PhD), The University of Texas at Austin, Texas.

Queiroz, C. A. V. (1984) Modelos de previsão de desempenho para a gerência de pavimentos no Brasil. Brasília: GEIPOT, 366 p.

Watanada, T., Paterson, W., Bhandi, A., Harral, C., Dhareshwar, A. M. and Tsunokawa, K. (1987) The Highway Design and Maintenance Standards Model. vol. 1. Description of de HDM III model. 